\title{
Oscillatory behaviour in Type IA FBG: ruling out chemical complexity
}

George Simpson, Kyriacos Kalli, John Canning, Amedee Lacraz

George Simpson, Kyriacos Kalli, John Canning, Amedee Lacraz, "Oscillatory behaviour in Type IA FBG: ruling out chemical complexity," Proc. SPIE 9634, 24th International Conference on Optical Fibre Sensors, 96345L (28 September 2015); doi: 10.1117/12.2195101

SPIE Event: International Conference on Optical Fibre Sensors (OFS24), 2015, Curitiba, Brazil 


\title{
Oscillatory behaviour in Type IA FBG: ruling out chemical complexity
}

\author{
George Simpson ${ }^{\mathrm{a}}$, Kyriacos Kalli * ${ }^{\mathrm{b}}$, John Canning ${ }^{\mathrm{c}}$ and Amedee Lacraz ${ }^{\mathrm{b}}$ \\ ${ }^{\mathrm{a}}$ Technology Consulting, Deloitte Touche Tohmatsu, Perth, Western Australia \\ ${ }^{\mathrm{b}}$ Nanophotonics Research Laboratory, Cyprus University of Technology, Limassol, Cyprus \\ ${ }^{c}$ interdisciplinary Photonics Laboratories, School of Chemistry, The University of Sydney, Australia
}

\begin{abstract}
Type IA FBG are regenerated gratings that appear in hydrogenated germanosilicate fibre of all types during prolonged UV exposure. The gratings are characterised by a large Bragg wavelength shift and a concomitant increase in the mean fibre core index. Modulated index changes are complex by comparison and significantly weaker, often characterised by oscillatory growth behaviour. Low thermal stability of Type IA gratings suggests a possible chemical role similar to thermally processed optical fibres where autocatalysis has been observed. We show that $\mathrm{GeOH}$ and $\mathrm{SiOH}$ formation are not out-of-phase and follow each other, with no evidence of autocatalysis, ruling out a chemical origin.
\end{abstract}

Keywords: fibre Bragg gratings, optical fibre sensors, absorption spectra, hydrogenation

\section{INTRODUCTION}

Type IA fibre Bragg gratings (FBG) are formed following the erasure of a Type I grating in hydrogenated germanosilicate fibre of all types at room temperature [1]. They are characterised by a large increase in the Bragg wavelength $\left(\lambda_{\text {Bragg }}\right)$ and a concomitant increase in the mean index $\left(\Delta n_{\text {eff }}\right)$ of the fibre core (Figure 1). Type IA gratings have also been designated Type $1 p$ because the index change is positive [2]. The modulated index change $\left(\Delta n_{\bmod }\right)$ is more complicated, and is characterised by oscillatory growth behaviour. The overall grating growth is correlated with absorption features that appear at $\sim 1400 \mathrm{~nm}$ and that can be deconvolved into the relevant $\mathrm{SiOH}$ and $\mathrm{GeOH}$ bands formed in the fibre. The material changes are localised and occur only in the region of UV laser exposure and are initiated by the combination of hydrogenation conditions and UV-laser fluence. This increase in $\Delta \lambda_{\text {Bragg }}$ is dependent on fibre type and the fibre hydrogen loading conditions [3]. In the case of highly doped fibres, such as pure Ge or B/Ge co-doped fibre, $\Delta \lambda_{\text {Bragg }} \sim 15-20 \mathrm{~nm}$, whereas for hydrogenated SMF28 it is lower at $\Delta \lambda_{\text {Bragg }} \sim 5-8 \mathrm{~nm}$ [3]. Such large values of $\Delta \lambda_{\text {Bragg }}$ translate to an effective core index increase up to $\Delta n_{\text {eff }} \sim 2 \times 10^{-2}$. We believe that these index modifications must reflect material changes within the core and core-cladding regions, consistent with net structural relaxation and therefore stress changes at the core-cladding interface. The extraordinarily large fluence involved further supports an annealing-like relaxation. Moreover, such a large increase in $\Delta n_{\text {eff }}$ raises the numerical aperture (NA) of the optical fibre so that it becomes few-moded [4] and the NA of the fibre can be increased in excess of 50\%. These changes are well beyond the local polarisability changes around altered or induced microscopic defect sites alone. Figure 2 shows the strong correlation between the growth of the $\mathrm{OH}$ absorption feature during prolonged UV exposure and the increase in $\Delta \lambda_{\text {Bragg. }}$. The absorption is characterised by the formation of both $\mathrm{SiOH}$ and $\mathrm{GeOH}$ groups; this becomes more complicated when other fibre dopants are considered. Given that the formation of these species can provide an indication of net energy imparted into the network, high temperature thermal annealing can lead to auto-oscillatory behaviour as hydrogen outdiffuses [5]. The failure to observe this in Type IA grating formation suggests insufficient effective temperatures induced by UV excitation; transient Bragg wavelength shifts during inscription show that it is between 100 and $200^{\circ} \mathrm{C}$. We may initially conclude that auto-oscillatory catalysis does not occur and that out-diffusion of hydrogen is relatively low - but is this supposition premature given the role of UV-induced bond breaking? The formation of $\mathrm{OH}$ in glass is strongly associated with a net local volume increase leading to relaxation of the tensile stresses at the core-cladding interface. However, volume expansion correlates with a negative index drop convolved with a positive index increase with stress relief leading to a positive index increase. The polarisability of $\mathrm{OH}$ has a strong positive index as well. The net result is a large $\Delta \lambda_{\text {Bragg }}$ shift arising from a large de index change at the core that is not reflected in the index modulation, indicating two different index origins.

* kyriacos.kalli@cut.ac.cy; phone+35725002609; www.cut.ac.cy 


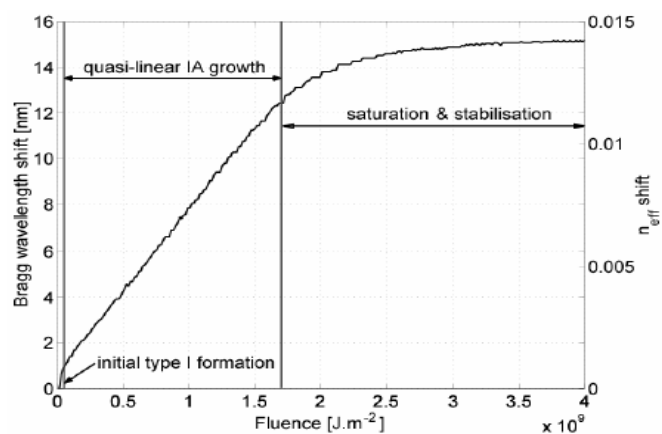

Figure 1. Characteristic red shift during prolonged UV exposure of hydrogenated germanosilicate fibre.

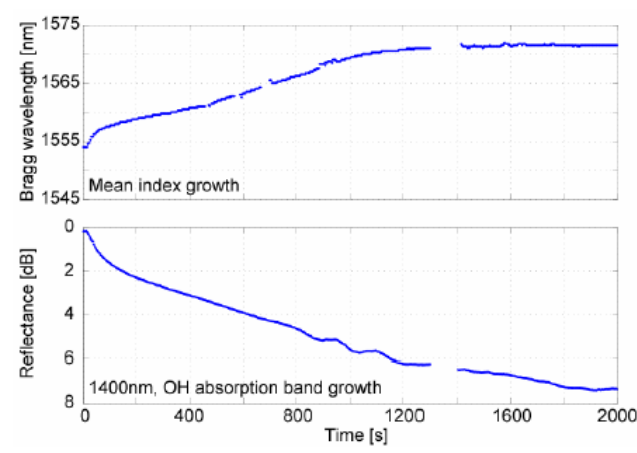

Figure 2. Mean index change (or IA maturity) and the $1400 \mathrm{~nm}$ absorption band under UV exposure.

So strong is the correlation between $\mathrm{OH}$ formation and $\Delta n_{\text {eff }}$ that by monitoring the growth of the $\mathrm{OH}$ absorption (Figure 2), one can determine whether the fibre has "matured" fully, and any gratings written will have the properties of Type IA FBG [4]. This is clear evidence that the dc changes and the grating properties themselves, whilst correlated, are unrelated in origin - the grating is "burnt" into the $\mathrm{OH}$ rich glass possibly through a shift of the $\mathrm{OH}$ distribution in the highs of the UV interferogram due to bond breaking and diffusion. We note that the peak absorption band loss at $\sim 1400 \mathrm{~nm}$ is typically $\sim 1.2 \mathrm{~dB} / \mathrm{mm}$ for a $\mathrm{B} / \mathrm{Ge}$ co-doped fibre. It is clear that there is a complicated process of chemical interaction occurring in the glass matrix that is driven by the presence of hydrogen and the UV-laser fluence. Indeed, it has been noted that autocatalysis and oscillatory reactions can occur in silicate glass systems under certain conditions where diffusion occurs [5]. We can rule this out because the induced temperatures are too low for diffusion between $\mathrm{Si}$ and $\mathrm{Ge}$ sites. A key result is the large structural change following the substantial formation of $\mathrm{SiOH}$ and $\mathrm{GeOH}$ sites in the highly Ge-doped fibre, where conditions may differ from those reported previously. Hence we address whether UV irradiation can lower the effective thermal environment required for autocatalysis. To explore this we undertake a study to either observe or rule out the presence of autocatalytic processes during Type IA grating inscription. By monitoring the increase in absorption at $1400 \mathrm{~nm}$, with increasing laser fluence, and decoupling the absorption features associated with the presence of $\mathrm{SiOH}$ and $\mathrm{GeOH}$, we look for coupling between the two bands in the form of out of phase wavelength oscillations [5]. The logic behind our investigation is summarised here:

(1) If oscillations are present and linked to coupling between $\mathrm{SiOH}$ and $\mathrm{GeOH}$, their respective absorption bands will appear out of phase, and follow an exponential decay profile because free $\mathrm{OH}$ or $\mathrm{H}$ will hop between sites and slowly diffuse out of the fibre core. For this to occur, there needs to be equivalence between $\mathrm{GeOH}$ and $\mathrm{SiOH}$ free energies, which can only be achieved when their free energy of formation is lower than the surrounding energy bath of the network; at room temperature this is impossible and temperatures above $500^{\circ} \mathrm{C}$ are necessary. Here the effective temperature is $\sim 100^{\circ} \mathrm{C}$, so we rely on UV bond breaking to reduce the energies of both Ge and Si sites to allow much lower phonon energies to release $\mathrm{OH}$ or $\mathrm{H}$, with index linked to differences between the index polarisability at the $\mathrm{GeOH}$ and $\mathrm{SiOH}$ sites.

(2) If we observe that the decoupled absorption features are oscillating in phase then a chemical origin for the observed index modulations is not possible, leading to other directions. Diffusion should also be observed via exponential decay.

\section{RESULTS AND DISCUSSION}

Gratings were inscribed using the scanning phase mask technique $\left(\Lambda_{\text {mask }}=1071.92 \mathrm{~nm}\right)$ and the fibre was illuminated with a CW UV Coherent Sabre FreD laser source operating at $\lambda=244 \mathrm{~nm}$. Laser powers of $200 \mathrm{~mW}$ were delivered to the fibres that were hydrogenated at $P=200$ bar pressure and $T=80^{\circ} \mathrm{C}$ for a 5-day period.

\subsection{OH band structure in different fibre types}

Gratings were manufactured using the fibre types described in Table 1;20-mm fibre sections were exposed to blankbeam UV radiation at a scan speed of $1 \mathrm{~mm} / \mathrm{s}$ and were monitored for transmission loss in the $\sim 1400 \mathrm{~nm}$ region. The $\mathrm{OH}$ bands formed in these different fibre types are shown in Figure 3(a). The traces show that B/Ge fibre (blue) and SMF28 
(cyan) both exhibit asymmetric Gaussian absorption peaks. The remaining plots show the $\mathrm{OH}$ bands formed in Ge-doped fibres (Verillion and Fibercore), showing two main peaks. All features are predominantly related to the relative contributions of $\mathrm{SiOH}$ and $\mathrm{GeOH}$.

\begin{tabular}{|c|c|c|c|c|c|c|c|c|}
\hline \multirow{2}{*}{ Fibre reference } & \multirow{2}{*}{ Manufacturer } & \multirow{2}{*}{$\begin{array}{l}\text { Figure } 3 \text { a colour } \\
\text { convention }\end{array}$} & \multirow{2}{*}{$\begin{array}{l}\text { Fibre } \\
\text { type }\end{array}$} & \multirow{2}{*}{ NA } & \multicolumn{2}{|c|}{ Main peak } & \multicolumn{2}{|c|}{ Secondary peak } \\
\hline & & & & & $\mathrm{nm}$ & $\mathrm{dB} / \mathrm{mm}$ & $\mathrm{nm}$ & $\mathrm{dB} / \mathrm{mm}$ \\
\hline SMF28 & Corning & Cyan & STD & 0.14 & 1388 & 0.51 & $\mathrm{U}$ & $\mathrm{U}$ \\
\hline PS1250/1500 & Fibercore & Blue & $\mathrm{B} / \mathrm{Ge}$ & 0.14 & 1404 & 1.22 & 1425 & $\mathrm{U}$ \\
\hline IF0100331001 & Verillion & Red-dashed & $\mathrm{Ge}$ & 0.23 & 1392 & 0.62 & 1406 & 0.51 \\
\hline SD079-00B & Fibercore & Green & $\mathrm{Ge}$ & 0.24 & 1393 & 0.68 & 1408 & 0.63 \\
\hline SD748A-00AB & Fibercore & Black & $\mathrm{Ge}$ & 0.26 & 1412 & 0.71 & 1397 & 0.66 \\
\hline SD254-01B & Fibercore & Yellow & $\mathrm{Ge}$ & 0.30 & 1412 & 0.72 & 1395 & 0.64 \\
\hline FA1631211 & Verillion & Magenta & $\mathrm{Ge}$ & 0.30 & 1414 & 0.76 & 1397 & 0.60 \\
\hline
\end{tabular}

Table 1. Summary of the absorption band peaks and dopant levels for the fibres tested. U - Unidentified.

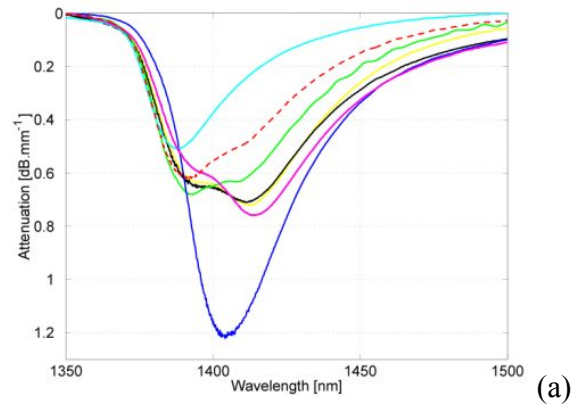

Figure 3. (a) $\mathrm{OH}$ bands created in the optical fibres listed in Table 1, by uniform exposure of 20 -mm fibre sections to UV radiation.

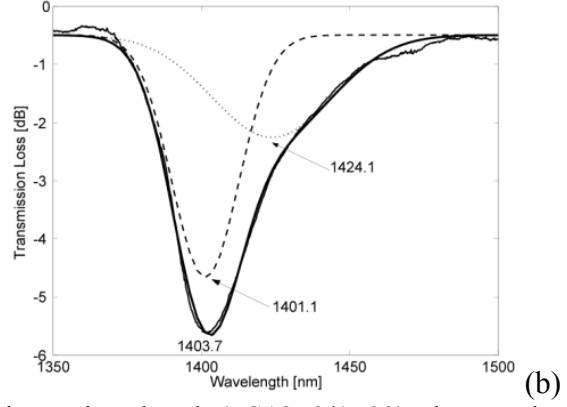

(b) 1400-nm absorption band (PS1250/1500) deconvolved to show the constituent absorption peaks. The anticipated mixture of $\mathrm{SiOH}(1390 \mathrm{~nm}) \&$ $\mathrm{GeOH}(1410 \mathrm{~nm})$ bands are shifted due to the presence of fibre dopants.

Deconvolution of the PS1250/1500 fibre data shows the presence of two peaks, Figure 3(b). Note that the peak of the curve is just beyond $1400 \mathrm{~nm}$ under continuous UV exposure, indicating that the anticipated contributions from a mixture of $\mathrm{SiOH}(1390 \mathrm{~nm})$ and $\mathrm{GeOH}(1410 \mathrm{~nm})$ bands are biased in favour of the $\mathrm{GeOH}$ bonds for this fibre; this is consistent with both lower free energy of formation for $\mathrm{GeOH}$ and the high concentration of $\mathrm{Ge}$ in the core. The solid trace represents the absorption band as measured with an optical spectrum analyser (OSA). The dashed and dotted traces represent the de-convolved Gaussian peaks within the absorption band and the bold solid trace shows the addition of these two traces, which closely follows the experimental data. The deconvolved peaks are located at $1401.1 \mathrm{~nm}$ (attributed to $\mathrm{SiOH}$ contribution) and $1424.1 \mathrm{~nm}$ (attributed to $\mathrm{GeOH}$ contribution). However, we note that the deconvolved peaks are shifted from the anticipated peaks at $1390 \mathrm{~nm}(\mathrm{SiOH})$ and $1410 \mathrm{~nm}(\mathrm{GeOH})$, which we believe results from the presence of $\mathrm{B}$ in the core. There is also no evidence of a hydride band at $1500 \mathrm{~nm}$ that is most often associated with $\mathrm{GeH}$; this may indicate the rapid conversion of the hydride to the more stable hydroxyl form, an indication of the underlying complexity involved with numerous routes to $\mathrm{OH}$ formation. If we monitor the absorption bands of selected fibres under prolonged UV exposure, we observe that there are significant spectral similarities between the different fibre types shown in Figure 4. The B/Ge co-doped fibres (Figure 4(a) and (b)) both show the same fundamental shape as deconvolved in Figure 3(b), whereas the three Ge-doped fibres show the presence of $\mathrm{SiOH}$ at $1390 \mathrm{~nm}$. By deconvolving the absorption features for the Fibercore fibre (SD079-00B), linked only to $\mathrm{SiOH}$ and $\mathrm{GeOH}$ bands, we evaluated the band peaks and areas with the laser scan number, Figure 5. It is clear that the curves display some form of oscillation but that it occurs in phase (Figure 5(a)), from which we can exclude case (1) in favour of case (2), suggesting that under our combined optical fibre/inscription conditions autocatalysis does not occur. Figure 5(a) shows the evolution of the peak wavelengths of the $\mathrm{SiOH}$ and $\mathrm{GeOH}$ bands with increasing exposure, showing an exponential decay profile as $\mathrm{H}$ diffuses out of the fibre core (Fibercore SD079-00B). These peak wavelengths asymptote to the anticipated $\mathrm{SiOH}$ and $\mathrm{GeOH}$ values with each scan, Figure 5(b). Equilibrium is reached less readily for $\mathrm{GeOH}$ than for $\mathrm{SiOH}$, which rapidly reaches saturation after 70 scans; this is consistent with there being less $\mathrm{SiOH}$ in the core region. Figure 5(c) confirms that there are no out-of-phase oscillations. 


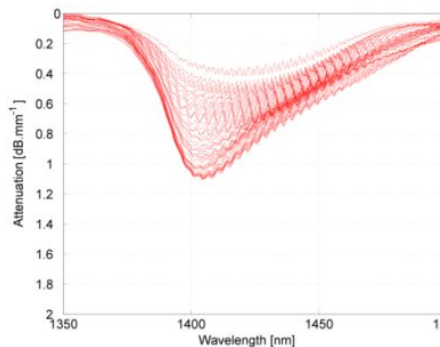

(a)

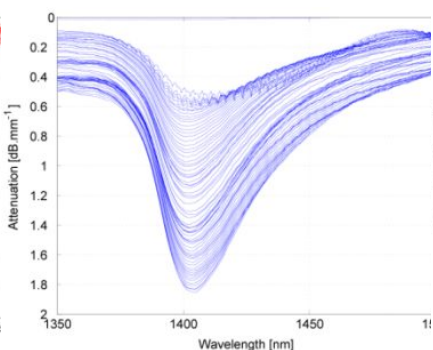

(b)

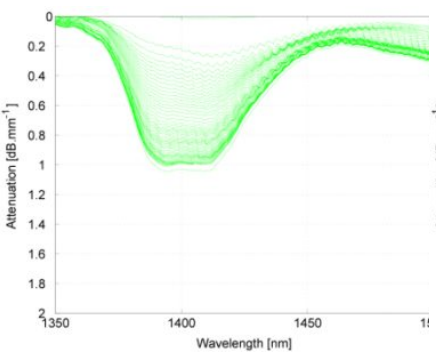

(c)

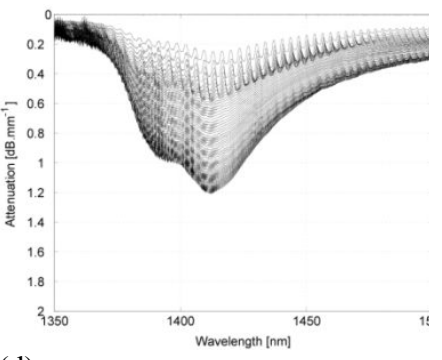

(d)

Figure 4. Evolution of the $\mathrm{OH}$ band associated with the formation of IA gratings during inscription in Verillion IF01001410101 B/Ge (a), Fibercore PS1250/1500 B/Ge (b), Fibercore SD079-00B Ge (c) and Fibercore SD748A-00AB Ge (d). This figure shows that the competing $\mathrm{OH}$ species have are weighted according to fibre dopants and doping levels.

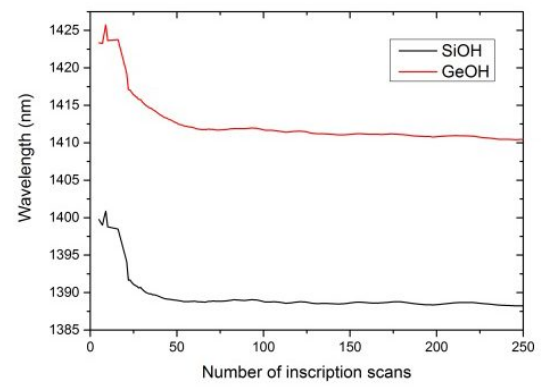

Figure 5. (a) Evolution of $\mathrm{SiOH}$ and $\mathrm{GeOH}$ absorption band peak wavelengths with laser scan number.

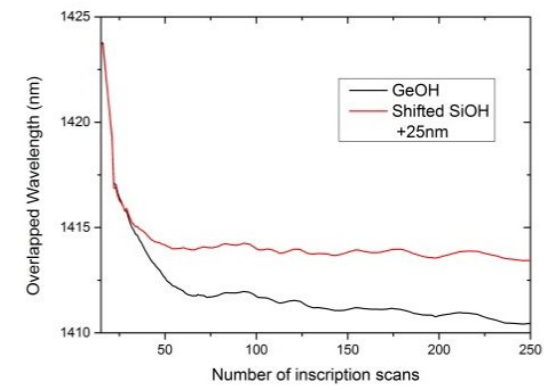

(b) Superimposed $\mathrm{SiOH} / \mathrm{GeOH}$ curves to compare growth and equilibrium with laser scan number.
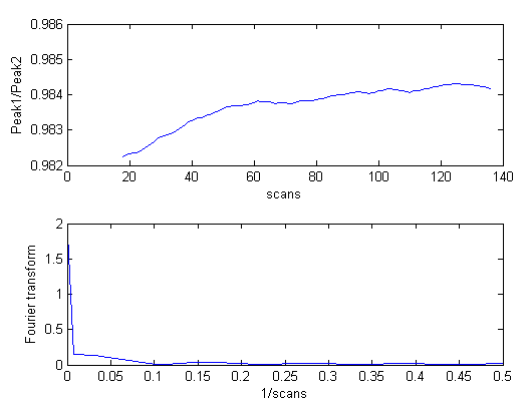

(c) Ratio of $\mathrm{SiOH} / \mathrm{GeOH}$ absorption band peak wavelengths with scan number and their FFT, excluding out-of-phase oscillations.

In conclusion, for Germanium doped fibre (Fibercore SD079-00B), we show that there is no autocatalysis process contributing to Type IA grating formation, despite the role of $\mathrm{OH}$ in the large dc shift and possible role as a photorefractive component generating the low thermal stability of the modulated index. This is consistent with a background energy being too low to equalise the energy levels of the general $\mathrm{GeO}$ and $\mathrm{SiO}$ bond sites for $\mathrm{OH}$ formation. The combined UV bond breaking and effective heating are unable to provide this energy. The net dc shift linked to Type IA gratings can effectively be described as part of the continuum of annealing behaviour possible in composite glass fibre systems, especially when $\mathrm{H}$ is present, and the role of chemical complexity in elucidating some of the finer details observed during grating growth processes is ruled out for the Type IA grating in this case - i.e. the observed index modulation is not a reflection of change between $\mathrm{OH}$ species indicating other sources for this.

\section{ACKNOWLEDGMENTS}

The views expressed in this paper do not represent an official position of Deloitte Touche Tohmatsu.

The experimental work, which has been reinterpreted here, was performed at Aston University's Photonics Research Group. We acknowledge their considerable contribution, the generous funding of the Engineering and Physical Research Council, Indigo Photonics and BAE Systems and the sound council of Professors Ian Bennion and Lin Zhang.

\section{REFERENCES}

[1] Simpson A.G., Kalli K., Zhou K., Zhang L., Bennion I., "Formation of type IA fibre Bragg gratings in germanosilicate optical fibre", Elect. Lett., 40(3), 163-164 (2004)

[2] Canning J., "Fibre gratings and devices for sensors and lasers", Lasers \& Photon. Rev., 2(4), 275-289 (2008)

[3] Kalli K., Simpson A.G., Zhou K., Zhang L., Bennion I., "Tailoring the temperature and strain coefficients of type I and type IA dual grating sensors - the impact of hydrogenation conditions", Meas. Sci. Technol., 17, 949-954 (2006)

[4] Simpson A.G., Kalli K., Zhou K., Zhang L., Bennion I., "Blank beam fabrication of regenerated type IA gratings", Meas. Sci. Technol., 15, 1665-1669 (2004)

[5] Canning J., Sørenson H.R., Kristensen M., "Solid-state autocatalysis and oscillatory reactions in silicate glass systems", Opt. Comm., 260, 595-600, (2006) 